help was the solicitor instructed by my trust to defend me in court.

Having read the paper by Dewar et al, I feel I would have benefited from some training for the consequences or the organisational procedures following suicide. I feel I should have had a mentor or a similar senior person to open up to.

Senior house officers are the most vulnerable doctors, owing to their relative inexperience and the fact that it is often the first time they come across suicide. There should be a better support network when something on these lines occurs.

B. S. Ashim, 6 Hasper Avenue, Withington, Manchester M20 1AX

\section{Experience of community treatment orders}

Sir: We wish to reply to Moncrieff \& Smyth's paper on community treatment orders (CTOs) (Psychiatric Bulletin, November 1999, 23, 644-646). We write as British psychiatrists working in Melbourne where CTOs have been in use since 1987. In our service 161 adults (1865 years) out of a total catchment area population of 225000 are subject to a CTO. CTOs are regarded as part of good clinical facilitating treatment in the least restrictive environment many lasting for only a few months after hospital admission.

The CTO enables the clinician to insist on clinic attendance and the patient's acceptance of oral or intra-muscular medication. If the patient refuses to comply then the CTO may be revoked and the patient admitted to hospital, usually for a very brief period. This ultimate sanction is rarely required. The CTO is only one part of a comprehensive biopsychosocial care plan. An order may alter the dynamics of care, but the clinicianpatient relationship is usually remarkably well preserved. In our clinical experience, CTOs are most helpful if some sort of therapeutic alliance has been established. This alliance can be continually developed and improved particularly by psychoeducation sessions

We dispute Moncrieff \& Smyth's surprising statement that CTOs would increase the amount of medication administered and, therefore, side-effects In our experience, doses as low as $20 \mathrm{mg}$ of flupenthixol depot monthly are sufficient to spare an insightless patient the indignity of regular compulsory admissions to hospital with all the extra medication and restrictions that these inevitably entail. A prolonged symptom-free period may demonstrate the benefits of psychiatric care to a person with a recurrent psychotic illness.

The introduction of CTOs in Victoria was an integral part of the state-wide development of community care. They are not now, and never were, intended to be a means by which psychiatry could control antisocial behaviour.

The efficacy of CTOs has not been established, but having practised with them and without them we feel that they have an important part to play in good clinical care. We agree with Burns (Psychiatric Bulletin, November 1999, 23 647-648) and wish to move the debate away from the issue of prevention of violence towards the provision of humane, community-based care.

*David Protheroe, Consultant Psychiatrist, Andrew Carroll, Consultant Psychiatrist, Northern Hospital, 185 Cooper Street, Epping, 3076 Australia

Sir: The issue of community treatment orders (CTOs) continues to create difference within the College, but Moncrieff \& Smyth (Psychiatric Bulletin, November 1999, 23, 644-646) have added nothing new to the debate. Rather, by drawing the issue away from the practical question of how to help a small group of vulnerable people, towards vaguely defined issues of social control, they may be doing psychiatry a disservice. They have been selective in their references, avoiding the many articles evaluating CTOs in other countries, for example the USA and Australia. Most of their arguments are as relevant to any kind of compulsory treatment as to that specifically located in the community.

The notion, furthermore, that it was "concern for patient rights that underscored the move away from the asylums" is only partially true. There were certainly concerns about institutionalisation, recognised by numerous hospital inquiries, but the impacts of modern medication and of the resource implications of an ageing real estate were just as powerful. The rising demand for medium secure unit beds reflects the way in which the asylums are returning, in another form, while the rising level of Mental Health Act sections reflects the distress of a number of chaotic, relapsing individuals for whom current community provision is simply inadequate.

One of us recalls a rather angry argument in the 1970s, about the introduction of car seat-belts, in which a senior physician suggested that it was a gross infringement of personal rights to be ordered to wear a belt. Yet the impact of a seatbelt law, in terms of reduced head injury and general morbidity and reduced mortality, has been immense. With the appropriate legal safeguards, the use of enhanced tribunals or other legal agencies to monitor community treatment, and reversion of psychiatrists to their proper role (as therapists rather than turnkeys), it would seem to us that history can inform the usefulness of the CTO approach.

We strongly concur with Burns (Psychiatric Bulletin, November 1999, 23, 647-648) that these orders are not about bad behaviour, but about therapy and treatment for people with limited insight who have a right to such treatment. The challenge to psychiatry lies in educating the public that untoward behaviour will always occur, even with CTOs in place. The problem with the language of 'social control' is that it makes it very easy to be a bad Samaritan.

*TrevorTurner, Consultant Psychiatrist, Mark Salter, Consultant Psychiatrist,

Mary Howlett, Consultant Psychiatrist,

Martin Deahl, Consultant Psychiatrist, Division of Psychiatry, East Wing 2nd Floor, Homerton Hospital Homerton Row, London E9 6SR

\section{Survey of supervised discharge of mentally ill people}

Sir: A postal survey was conducted (April 1996-June 1997) to determine the attitudes of consultant psychiatrists working with adult, adolescent or psychogeriatric patients in the Wales Region towards the new legal powers.

After 12 weeks there had been a $31 \%$ (107/300) response rate. Only six patients in total were placed on supervised discharge by three of the 107 respondents. Twenty-eight of the 107 respondents stated that they had considered using the new legislation regarding supervised discharge, although 53 had reservations.

Three aspects caused concern when the respondents were asked about the factors that influenced the decision to recommend supervised discharge. Supervised discharge would generate an increased workload, for which resources are not available (5/107); there was no sanction on the patient if he or she did not comply (30/107); and, properly resourced community care could be used instead of supervised discharge (27/107). Several respondents commented that the legislation was insufficient in its powers and that the legislation would not be appropriate for patients lacking insight.

Respondents felt that confusion existed while processing the paperwork and the forms need to be simplified and clarified in order to avoid unnecessary time consumption.

Although the Act introduces a new somewhat convoluted system of procedures for supervised aftercare, and has serious implications for both human rights and the relationship between care professionals and their service users, it is difficult to see it as an improvement on the possibilities for intervention under 